\title{
Solving Diophantine equations with binomial coefficients in study group sessions using both elementary and higher mathematical methods
}

\author{
RICHÁRD RAKAMAZI
}

\begin{abstract}
The paper can be considered as the continuation of [4] in the sense that we are studying Diophantine equations containing binomial coefficients. It was an important aspect that one should be able to discuss these problems - even if not in complete depth - also in high school study group sessions with the most talented students. We present various methods through several examples, which help the successful handling of other questions too, including problems in math competitions. Our discussion starts with the elementary treatment of easier problems, and then proceed gradually to more difficult questions which require higher mathematical methods.
\end{abstract}

Key words and phrases: Diophantine equations, binomial coefficients, arithmetic, quadratic form.

ZDM Subject Classification: F60.

The primary purpose of the paper is to demonstrate how the solution of some special Diophantine equations by a wide range of methods can lead us from relatively simple questions to profound and complicated mathematical problems. These methods fit very well into the workshop activity of study group sessions from several points of view: They greatly enhance the students ${ }^{6}$ arsenal of mathematical methods, enable the efficient investigation of various difficulty levels (infinitely many solutions, finding all solutions), and introduce the gifted students into the art of conjecturing and proving by themselves. Thus the paper is 
strongly connected with those researches in mathematical didactics which examine the development of the cognitive abilities of students during the teaching and learning process explicitly within the Diophantine topic (see [9]).

Problem 1. Show that the Diophantine equation

$$
\left(\begin{array}{l}
x \\
2
\end{array}\right)+\left(\begin{array}{l}
y \\
2
\end{array}\right)=\left(\begin{array}{l}
z \\
2
\end{array}\right)
$$

has infinitely many solutions, where $x, y, z \geq 2, x, y, z \in \mathbb{Z}$.

Solution. If we expand the binomial coefficients and multiply the equation by 2 , we get

$$
x^{2}+y^{2}-x-y=z^{2}-z .
$$

After rearranging the equation and applying the possible factorization, we have

$$
x(x-1)=(z-y)(z+y-1) .
$$

Let $r=\operatorname{gcd}(x, z-y)$, thus $x=r s$ and $z-y=r t$, where $\operatorname{gcd}(s, t)=1$. We obtain that $s(x-1)=t(z+y-1)$. Since $\operatorname{gcd}(s, t)=1$, we have $z+y-1=s u$ and $x-1=t u$. From these equations we obtain

$$
x=r s, \quad y=\frac{u s-r t+1}{2}, \quad z=\frac{r t+u s+1}{2},
$$

where $r s-t u=1$. It is obvious that $y$ and $z$ are integers iff $u s$ and $r t$ are of opposite parity. The relation $r s-t u=1$ gives that $r s$ and $t u$ are of opposite parity, combining these we immediately obtain that $y$ and $z$ are integers iff exactly one of $r, s, u, t$ is even. For example, if $r=1, s=4(k+1)^{2}, u=2 k+3$ and $t=2 k+1$, where $k \in \mathbb{N}$ the relevant values $x, y, z$ are

$$
x=4(k+1)^{2}, \quad y=4 k^{3}+14 k^{2}+15 k+6, \quad z=4 k^{3}+14 k^{2}+17 k+7 .
$$

Problem 2. Show that the Diophantine equation

$$
\left(\begin{array}{c}
x-1 \\
2
\end{array}\right)+\left(\begin{array}{l}
x \\
2
\end{array}\right)+\left(\begin{array}{c}
x+1 \\
2
\end{array}\right)=\left(\begin{array}{l}
y \\
2
\end{array}\right)
$$

has infinitely many solutions, where $x \geq 3, y \geq 2, x, y \in \mathbb{Z}$.

Solution. If we expand the binomial coefficients and apply the possible cancellations we have

$$
3 x^{2}-3 x+2=y^{2}-y .
$$


After multiplying both sides by 4 we can form complete squares:

$$
\begin{aligned}
& 12 x^{2}-12 x+8=4 y^{2}-4 y, \\
& 3(2 x-1)^{2}+6=(2 y-1)^{2} .
\end{aligned}
$$

Putting $v=2 x-1$ and $u=2 y-1, u, v \in \mathbb{Z}$ we have

$$
u^{2}-3 v^{2}=6
$$

We show that this equation has infinitely many odd integer solutions $u, v$. Obviously, in any integer solution $u, v$ are odd integers (and thus $x$ and $y$ are integers), since if exactly one of $u$ and $v$ is even, then the LHS is odd and if both are even, then the LHS is divisible by 4.

Let us consider the following decomposition:

$$
(3-\sqrt{3})(3+\sqrt{3})=6
$$

and the so-called Pell's equation $s^{2}-3 r^{2}=1$ (see [6]). We easily get the values $s=2, r=1$, and this yields the decomposition $(2-\sqrt{3})(2+\sqrt{3})=1$. If we raise both sides of this equality to the $n$th power, then we have with suitable integers $\alpha_{n}, \beta_{n}$

$$
\left(\alpha_{n}-\sqrt{3} \beta_{n}\right)\left(\alpha_{n}+\sqrt{3} \beta_{n}\right)=1 .
$$

If we multiply this with (2) and expand the brackets, we get a new solution of equation (1). Let us illustrate this in the case $n=1$. We obtain

$$
(2-\sqrt{3})(2+\sqrt{3}) \cdot(3-\sqrt{3})(3+\sqrt{3})=(9-5 \sqrt{3})(9+5 \sqrt{3})=9^{2}-5^{2} \cdot 3=6 .
$$

Thus, $u=9$ and $v=5$ give a solution of equation (1). The relevant values $x, y$ are $x=3$ and $y=5$.

$$
\left(\begin{array}{l}
2 \\
2
\end{array}\right)+\left(\begin{array}{l}
3 \\
2
\end{array}\right)+\left(\begin{array}{l}
4 \\
2
\end{array}\right)=\left(\begin{array}{l}
5 \\
2
\end{array}\right)
$$

Problem 3. Show that the Diophantine equation

$$
\frac{\left(\begin{array}{l}
x \\
2
\end{array}\right)+\left(\begin{array}{l}
y \\
2
\end{array}\right)}{2}=z^{2}
$$

has infinitely many solutions, where $x \neq y$ and $x, y \geq 2, x, y, z \in \mathbb{Z}$.

Solution. Note that the equation has infinitely many solutions $x=y \geq 2$, since with equivalent conversions we get a Pell's equation (see below), which, 
as previously mentioned, has infinitely many solutions in positive integers. The equation is equivalent to

$$
x^{2}-x+y^{2}-y=(2 z)^{2} .
$$

After multiplying both sides by 4 , and forming complete squares we have

$$
(2 x-1)^{2}+(2 y-1)^{2}=(4 z)^{2}+2 .
$$

REMARK 4. The equation $A^{2}+B^{2}=C^{2}+2$ is a special Diophantine equation from the theory of ternary quadratic forms. In $1916 \mathrm{~S}$. Ramanujan investigated those integers which cannot be represented in the form $a x^{2}+b y^{2}+c z^{2}$ for certain specific values of $a, b$ and $c$. One of the most famous example is $x^{2}+y^{2}+10 z^{2}$. (See [8]).

For the time being we are not aiming to find all the integer solutions of the equation (Lemma 9), we are rather seeking infinitely many solutions for specific given conditions. It is a general truth, that even if there are too many variables, it is worth to decrease the number of the unknowns by a simple connection between the variables, if possible. In this case, by the substitution $B=C-1$ we get a diophantine equation that can be handled by elemental tools.

Let us consider the Diophantine equation $A^{2}+B^{2}=C^{2}+2$ and examine the equation modulo 8 . It is obvious, that $A, B>0$ are odd, $C>0$ and $4 \mid C$. Let us look for solutions with condition $B=C-1$. After applying the possible simplifications we have $A^{2}=2 C+1$. If we choose the value of $C$ as $2 n^{2}+2 n$, then $A=2 n+1$ and $B=2 n^{2}+2 n-1$, where $n>1$ is an integer. If we substitute these values, we get infinitely many solutions in integers $x, y, z$ :

$$
x=n+1, \quad y=n^{2}+n, \quad z=\frac{n(n+1)}{2} .
$$

REMARK 5. In general the equation $A^{2}+B^{2}=C^{2}+c$, where $c$ is a fixed integer has infinitely many solutions in integers. We get the following solutions after the substitution $B=C-1$ and simplification:

$$
A=t, \quad B=\frac{t^{2}-1-c}{2}, \quad C=\frac{t^{2}+1-c}{2} .
$$

Since the numerators of $B$ and $C$ have the same parity, we only need to require $t>0$ to be odd if $c$ is even, and to be even if $c$ is odd. 
REMARK 6. In the previous problem we have successfully given infinitely many integer solutions for the Diophantine equation $A^{2}+B^{2}=C^{2}+2$. This method can be taught at a high school level. Now, with the help of higher mathematical methods, we can give all integer solutions of the equation. For the deduction we use the relevant sections in book [2] related to the quadratic forms. We summarize the main definitions and theorems from the book.

\section{DEFinition 7.}

(i) The quadratic forms $f(x, y)=a x^{2}+b x y+c y^{2}$ and $g(x, y)=A x^{2}+B x y+C y^{2}$ are equivalent, if there exists a $2 \times 2$ matrix $M$ with integer entries, such that $\operatorname{det} M=1$, and $g(x, y)=f\left(m_{11} x+m_{12} y, m_{21} x+m_{22} y\right)$ for all integers $x, y$. Notation: $f \sim g$.

(ii) Let $f$ be a quadratic form whose discriminant $d$ is not a perfect square. We call $f$ reduced if

$$
-|a|<b \leq|a|<|c| \text { or } \quad 0 \leq b \leq|a|=|c| .
$$

\section{THEOREM 8.}

(i) Let $d$ be a given integer, which is not a perfect square. Each equivalence class contains at least one reduced quadratic form, whose discriminant is $d$.

(ii) Let $f$ be a reduced quadratic form. If $f$ is indefinite, then $0<|a| \leq \frac{\sqrt{d}}{2}$.

After all these preparations, the following lemma can be proved.

Lemma 9. The Diophantine equation $A^{2}+B^{2}=C^{2}+2$ has infinitely many solutions in integers, all integer solutions are given by

$$
\begin{gathered}
A=\frac{m_{11}^{2}-m_{12}^{2}-2 m_{21}^{2}+2 m_{22}^{2}}{2}, \quad B=m_{11} m_{12}-2 m_{21} m_{22}, \\
C=\frac{m_{11}^{2}+m_{12}^{2}-2 m_{21}^{2}-2 m_{22}^{2}}{2},
\end{gathered}
$$

where $m_{i j}$ are integers, $m_{11}, m_{12}$ are odd integers and $m_{11} m_{22}-m_{12} m_{21}=1$. In the statement, the roles of $A$ and $B$ are interchangeable.

Proof. Let us consider the equation $A^{2}+B^{2}=C^{2}+2$ modulo 8. It is obvious, that we can get integer solutions only if $A, B$ are odd, and $4 \mid C$. Therefore there exist odd numbers $a, b$ and $c$, that for example $A=\frac{a-c}{2}, B=b$ and $C=\frac{a+c}{2}$. After substitution we obtain $4 b^{2}-4 a c=8$ which is exactly 
the discriminant of the quadratic form $f(X, Y)=a X^{2}+2 b X Y+c Y^{2}$, where $d=8$. Based on Definition 7 and Theorem 8 , there exists a reduced quadratic form $g(X, Y)=a^{\prime} X^{2}+2 b^{\prime} X Y+c^{\prime} Y^{2}$ equivalent to $f$, with $d^{\prime}=8,\left|a^{\prime}\right| \leq\left|c^{\prime}\right|$, $\left|2 b^{\prime}\right| \leq\left|a^{\prime}\right|$ and $0<\left|a^{\prime}\right| \leq \frac{\sqrt{8}}{2}$. According to the last condition we get $\left|a^{\prime}\right|=1$, thus $a^{\prime}=1$ or $a^{\prime}=-1$. In both cases only $b^{\prime}=0$ is possible. Substituting this into $\left(2 b^{\prime}\right)^{2}-4 a^{\prime} c^{\prime}=8$, we have $c^{\prime}=-2$ if $a^{\prime}=1$, and $c^{\prime}=2$ if $a^{\prime}=-1$. The two reduced quadratic forms are $g(X, Y)=X^{2}-2 Y^{2}$ and $g^{\prime}(X, Y)=-X^{2}+2 Y^{2}$. It is enough to consider one of them, since the two quadratic forms are equivalent, as based on Definition 7 the matrix

$$
M=\left(\begin{array}{ll}
1 & -2 \\
1 & -1
\end{array}\right)
$$

will be appropriate. Since $f \sim g$, there exist integers $m_{11}, m_{12}, m_{21}, m_{22}$ such that

$$
a X^{2}+2 b X Y+c Y^{2}=\left(m_{11} X+m_{12} Y\right)^{2}-2\left(m_{21} X+m_{22} Y\right)^{2}
$$

for all integers $X, Y$ and $\operatorname{det} M=1$, i.e. $m_{11} m_{22}-m_{12} m_{21}=1$. After expanding the brackets and comparing the coefficients we have

$$
m_{11}^{2}-2 m_{21}^{2}=a, \quad m_{11} m_{12}-2 m_{21} m_{22}=b, \quad m_{12}^{2}-2 m_{22}^{2}=c .
$$

This gives

$$
\begin{gathered}
A=\frac{m_{11}^{2}-m_{12}^{2}-2 m_{21}^{2}+2 m_{22}^{2}}{2}, \quad B=m_{11} m_{12}-2 m_{21} m_{22}, \\
C=\frac{m_{11}^{2}+m_{12}^{2}-2 m_{21}^{2}-2 m_{22}^{2}}{2} .
\end{gathered}
$$

Here $A$ and $C$ can be integers only if $m_{11}$ and $m_{12}$ have the same parity, but they cannot be both even, since in this case the condition $m_{11} m_{22}-m_{12} m_{21}=1$ cannot be satisfied. Let us consider e.g. $m_{11}=5, m_{22}=3, m_{12}=7$ and $m_{21}=2$. After substitution we get the values $A=-7, B=23$ and $C=24$ for which $(-7)^{2}+23^{2}=24^{2}+2$.

With the help of Lemma 9, we can give all solutions of equation $\frac{\left(\begin{array}{l}x \\ 2\end{array}\right)+\left(\begin{array}{l}y \\ 2\end{array}\right)}{2}=z^{2}$ :

$$
\begin{aligned}
x & =\frac{m_{11}^{2}-m_{12}^{2}-2 m_{21}^{2}+2 m_{22}^{2}+2}{4} \\
y & =\frac{m_{11} m_{12}-2 m_{21} m_{22}+1}{2} \\
z & =\frac{m_{11}^{2}+m_{12}^{2}-2 m_{21}^{2}-2 m_{22}^{2}}{8} .
\end{aligned}
$$


Here $x, y$ and $z$ are integers iff $m_{11}$ and $m_{12}$ are both odd, exactly one of $m_{21}$ and $m_{22}$ is even and $m_{11} m_{22}-m_{12} m_{21}=1$. Further $x, y, z \geq 2$, and $x$ and $y$ are interchangeable. Let us consider e.g. $m_{11}=7, m_{22}=0, m_{12}=1$ and $m_{21}=-1$ : after substitution we have $x=12, y=4$ and $z=6$ and get $\frac{\left(\begin{array}{c}12 \\ 2\end{array}\right)+\left(\begin{array}{c}4 \\ 2\end{array}\right)}{2}=6^{2}$.

As a special case, $m_{11}=2 n+1, m_{12}=1, m_{21}=-1$ and $m_{22}=0$ yield the solutions found in Problem 3.

Problem 10. Show that the Diophantine equation

$$
\left(\begin{array}{l}
x \\
2
\end{array}\right)+\left(\begin{array}{l}
y \\
2
\end{array}\right)=z^{2}
$$

has infinitely many solutions, where $x, y \geq 2, x, y, z \in \mathbb{Z}$.

We start with presenting various elementary arguments giving certain types of infinitely many solutions, and finally describe all integer solutions.

Solution 1. If we substitute $y=x+1$ and expand the binomial coefficients we obtain:

$$
\left(\begin{array}{c}
x \\
2
\end{array}\right)+\left(\begin{array}{c}
x+1 \\
2
\end{array}\right)=\frac{x(x-1)}{2}+\frac{(x+1) x}{2}=x^{2} .
$$

Hence the triple $(x ; x+1 ; x)$ is a solution for any $x \geq 2$.

Solution 2. We can get the same result from the following equivalent form of the equation:

$$
(2 x-1)^{2}+(2 y-1)^{2}=2(2 z)^{2}+2 .
$$

Considering the Diophantine equation $A^{2}+B^{2}=2 C^{2}+2$, clearly $A=C-1$, $B=C+1$ is a solution.

This gives

$$
x=\frac{t}{2}, \quad y=\frac{t+2}{2}, \quad z=\frac{t}{2},
$$

where $t \geq 1$ is an arbitrary even number.

Solution 3. Improving this method we can find infinitely many integer solutions, where $x, y, z$ are pairwise distinct. Let us consider the equation $A^{2}+$ $B^{2}-2 C^{2}=2$, where $A, B$ are odd integers and $C$ is even. Since $2^{2}+14^{2}-2 \cdot 10^{2}=$ 0 , we look for solutions of the form

$$
(2 n+\alpha)^{2}+(14 n+\beta)^{2}-2(10 n+\gamma)^{2}=2,
$$


where $n \geq 1$ is an integer, and $\alpha, \beta, \gamma$ are properly chosen parameters. After cancellation and comparing the coefficients on both sides we obtain $\alpha^{2}+\beta^{2}-2 \gamma^{2}=$ 2 and $\alpha+7 \beta-10 \gamma=0$. Substituting $\alpha=10 \gamma-7 \beta$ into the first relation we get $(5 \beta-7 \gamma)^{2}=1$, so for example $\beta=3, \gamma=2, \alpha=-1$ are suitable. Hence $(2 n-1)^{2}+(14 n+3)^{2}=2(10 n+2)^{2}+2$ holds, and we get

$$
x=n, \quad y=7 n+2, \quad z=5 n+1 .
$$

Solution 4 (All integer solutions of the equation). Let us rewrite the equation $(2 x-1)^{2}+(2 y-1)^{2}=2(2 z)^{2}+2$ in the form

$$
(y-x)^{2}+(x+y-1)^{2}=(2 z)^{2}+1 .
$$

After rearranging and factorizing we obtain

$$
(x+y-2)(x+y)=(2 z-y+x)(2 z+y-x) .
$$

As seen in the solution of Problem 1, this implies that there exist integers $r, s, t, u$ for which

$$
\begin{aligned}
x+y-2 & =r s \\
x+y & =t u \\
2 z-y+x & =r t \\
2 z+y-x & =s u .
\end{aligned}
$$

From these we have

$$
\begin{aligned}
x & =\frac{2 t u+r t-s u}{4} \\
y & =\frac{2 t u-r t+s u}{4} \\
z & =\frac{r t+s u}{4} .
\end{aligned}
$$


Furthermore, the condition tu $-r s=2$ must hold and the following table contains the possible remainders of the integers $t, u, r, s$ modulo 4 :

\begin{tabular}{|c|c|c|c|c|c|c|c|c|}
\hline $\boldsymbol{t}$ & $\boldsymbol{u}$ & $\boldsymbol{r}$ & $\mathbf{s}$ & & $\boldsymbol{t}$ & $\boldsymbol{u}$ & $\boldsymbol{r}$ & $\boldsymbol{s}$ \\
\hline 3 & 1 & 1 & 1 & & 1 & 2 & 0 & 2 \\
1 & 3 & 1 & 1 & & 2 & 1 & 2 & 0 \\
1 & 1 & 3 & 1 & & 0 & 2 & 1 & 2 \\
1 & 1 & 1 & 3 & & 2 & 0 & 2 & 1 \\
3 & 1 & 3 & 3 & 3 & 2 & 0 & 0 \\
1 & 3 & 3 & 3 & 2 & 3 & 0 & 0 \\
3 & 3 & 3 & 1 & 0 & 0 & 3 & 2 \\
3 & 3 & 1 & 3 & 0 & 0 & 2 & 3 \\
1 & 2 & 0 & 0 & 3 & 2 & 0 & 2 \\
2 & 1 & 0 & 0 & 2 & 3 & 2 & 0 \\
0 & 0 & 1 & 2 & 0 & 2 & 3 & 2 \\
0 & 0 & 2 & 1 & 2 & 0 & 2 & 3 \\
\hline
\end{tabular}

As special cases, $t=1, u=2 m+1, r=2 m-1$ and $s=1(m \in \mathbb{Z})$ give the triples obtained in Solutions 1 and 2, and $t=2, u=4 n+1, r=2 n$ and $s=4$ yield the result of Solution 3 . $z^{2}$.

As a related problem, finally we examine the Diophantine equation $\left(\begin{array}{l}x \\ 4\end{array}\right)+\left(\begin{array}{l}y \\ 4\end{array}\right)=$

Problem 11. Show that the Diophantine equation

$$
\left(\begin{array}{l}
x \\
4
\end{array}\right)+\left(\begin{array}{c}
x+1 \\
4
\end{array}\right)=y^{2}
$$

has infinitely many solutions, where $x \geq 4, x, y \in \mathbb{Z}$.

Solution. Expanding the terms on the LHS of the equation we obtain

$$
x(x-1)(x-2)(x-3)+(x+1) x(x-1)(x-2)=24 y^{2} .
$$

After applying the possible cancellations we get

$$
x(x-2)(x-1)^{2}=12 y^{2}=3(2 y)^{2} .
$$

The factor $x(x-2)$ on the LHS of the equation is relatively prime to $(x-1)^{2}$, therefore the equality holds iff $x(x-2)=3 u^{2}$ for a suitable integer $u$. This last 
equation can be written in the form $(x-1)^{2}-3 u^{2}=1$. Here we use again the theorem about Pell's equation (another equivalent shape), which can be proven with the argument seen in Problem 2: If $\left(\alpha_{0}, \beta_{0}\right)$ is a non-trivial solution of $\alpha^{2}-3 \beta^{2}=1$, then we get infinitely many integer solutions $\left(\alpha_{n}, \beta_{n}\right)$ of the form

$$
\alpha_{n}=\frac{\left(\alpha_{0}+\sqrt{3} \beta_{0}\right)^{n}+\left(\alpha_{0}-\sqrt{3} \beta_{0}\right)^{n}}{2}
$$

and

$$
\beta_{n}=\frac{\left(\alpha_{0}+\sqrt{3} \beta_{0}\right)^{n}-\left(\alpha_{0}-\sqrt{3} \beta_{0}\right)^{n}}{2 \sqrt{3}} .
$$

In our case $\alpha_{0}=2$ and $\beta_{0}=1$, so the formulas

$$
\alpha_{n}=\frac{(2+\sqrt{3})^{n}+(2-\sqrt{3})^{n}}{2}, \quad \beta_{n}=\frac{(2+\sqrt{3})^{n}-(2-\sqrt{3})^{n}}{2 \sqrt{3}} .
$$

give infinitely many solutions.

For example $n=3$ implies $\alpha_{3}=26$, from which we get $x=27$. In fact,

$$
\left(\begin{array}{c}
27 \\
4
\end{array}\right)+\left(\begin{array}{c}
28 \\
4
\end{array}\right)=195^{2} .
$$

\section{Didactical analysis, conception}

Teaching in a secondary school (not in a class specialized in maths) we generally deal with Diophantine equations in 2-3 ninety minute workshop sessions in a study circle. Of course, this may slightly vary according to the participants' mathematical interests and the teacher's personal preference. Fortunately, some excellent books and exercise collections ([1], [3]) are available for the teaching of Diophantine equations, and also an increasing number of Diophantine problems occur in the Hungarian maths competitions which serve as a good basis for the investigation of this topic outside the framework of the compulsory curriculum. In the paper we make an attempt to introduce some new types of interesting problems not forming part of the usual Diophantine agenda (linear equations, pythagorean triples, and proving the unsolvability via remainders with respect to a suitable modulus) which may be challenging for the best students.

These problems, similarly to the ones appearing in [4], are related to the binomial coefficients, and can thus greatly help the elaboration of this important mathematical notion. They can also help students to experience their own "discoveries" and to examine some further arithmetic connections of binomial coefficients. The author strongly believes in the importance of encouraging students 
to study literature and to do some sort of research in the topic (under appropriate supervision of a tutor, of course).

When compliling the exercises of the paper — in addition to the issues above - we tried to pay attention to the gradual approach, as well. In Problem 1 the method of factorization occurs (which is well known by the students), and then (and also in Solution 4 of Problem 10) the so-called four number theorem (see [7]) is suggested which — despite of its simplicity — can be well used to solve several problems of competitions (see [1]), and makes possible some elegant proofs during the workshops.

Problem 2 (similarly to Problem 11) illustrates well that sometimes we have to be contented with establishing an infinite number of non-trivial integer solutions, since finding all solutions is beyond our reach, at least in highschool. The handling of Pell's equation which we discuss here in detail, demonstrates in an excellent way that though the equation deals solely with integers, the natural method to treat it is to use real numbers, and to return to the integers only when exhibiting the solutions. This can be an ideal preparation for the discussion of "casus irreducibilis" in connection with cubic equations, where all the coefficients and roots are real numbers, but in order to establish these roots we have to make use of complex numbers.

Problem 3, and especially Problem 10 show that sometimes it is worth to start with simpler questions, because the solution of special cases is often much easier. It is a typical example that prescribing some simple relations for the variables still allows us to find infinitely many non-trivial solutions. Moreover, in certain cases, this way we can establish different types of solutions using substantially different parametrical forms. To arrive at the general solution, as it is shown in Lemma 7, sometimes we have to go beyond the secondary school curriculum. Quadratic forms have further profound and complicated applications (see [2]), we mention here just two of them: which integers can be represented as certain special forms (see the remark above), and how can we characterize the (prime) divisors of numbers of the form, say, $a^{2}+2 b^{2}$. The topic is worth to be considered, because in several cases these theorems facilitate the proof of the fact that a specific Diophantine equation has no solutions.

At this point - with very good students at hand - we can allow ourselves to have a glimpse at some more complicated equations, for example using Gaussianintegers (see [5]). Of course, at this level we have to give up the precise proofs covering all cases, but using a few theorems from higher mathematics we can demonstrate some interesting and beautiful statements to the students. (For 
example: Fermat's Christmas Theorem, and even the general result about which numbers are the sum of two squares, or new proofs or generalization of exercises solved earlier, e.g. in $E(\sqrt{-2}))$.

As a summary, we can state that another benefit of teaching Diophantine topic lies in showing the students how to find connections between various problems, how to combine different methods, how to generalize, how to ask new questions, even if some of them are beyond our proving capacities. During this teaching process, the teachers themselves learn a lot, and develop their own new methods, which is indispensable for all colleagues working with talented students.

\section{References}

[1] T. Andreescu, D. Andrica and I. Cucurezeanu, An Introduction to Diophantine Equations, Springer, 2010.

[2] I. Niven, H. S. Zuckerman and H. L. Montgomery, An Introduction to the Theory of Numbers, fifth ed., John Wiley \& Sons Inc., New York, 1991, 150-161.

[3] S. Róka, 2000 Problems In Elementary Mathematics, fifth ed., Typotex, Budapest, 2006, 29-33 (in Hungarian).

[4] R. Rakamazi, Diophantine equations concerning various means of binomial coefficients, Teaching Mathematics and Computer Science 12, no. 1 (2014), 71-79.

[5] R. Rakamazi, Solving Diophantine equations with elementary methods and with the help of Gaussian integers in high school mathematics study group session, Problem Solving in Mathematics Education (2013), 163-175.

[6] W. Sierpínski, Elementary Theory of Numbers, Polish Scientific Publishers, Warsaw, 1988, 88-100.

[7] P. Erdős and J. Surányi, Topics in the Theory of Numbers, Springer, 2003, 13-14.

[8] K. Ono and K. Soundararajan, Ramanujan's ternary quadratic form, Inventiones Mathematicae (1997), 415-454.

[9] L. Costica, Methods of solving Diophantine equations in secondary education in Romania, Science Journal of Education 2, no. 1 (2014), 22-32.

RICHÁRD RAKAMAZI

BAÁR-MADAS SECONDARY SCHOOL

BUDAPEST, HUNGARY

E-mail: raka82@gmail.com

(Received May, 2015) 